\title{
Medindo o preconceito racial no Brasil: Aniela Ginsberg e o estudo das atitudes raciais
}

Marcos Chor Maio*1

Este artigo aborda um conjunto de trabalhos realizados por Ginsberg sobre as relações étnico-raciais no Brasil. Procuro demonstrar que o processo de inflexão da visão de Ginsberg centrado inicialmente em investigações de natureza comportamental passa gradativamente a mobilizar argumentos socioantropológicos para o entendimento das assimetrias raciais. Argumento que tal mudança decorreu sobretudo da influência dos estudos do psicólogo social e antropólogo Otto Klineberg, da Universidade de Columbia, ex-orientando de Franz Boas, professor do Departamento de Psicologia da USP entre 1945 e 1947, e severo crítico dos testes de inteligência e do valor heurístico do conceito de raça.

Palavras-chave: Aniela Ginsberg, raça, racismo, testes de inteligência, atitudes raciais, Unesco

${ }^{1}$ Casa de Oswaldo Cruz/Fiocruz. (Rio de Janeiro, RJ, Br). 
A psicóloga Aniela Ginsberg exerceu papel de relevo na conformação de uma perspectiva sociopsicológica sobre as relações raciais no Brasil. Ao lado da socióloga e psicanalista Virginia Leone Bicudo (Maio, 2010), enfocou o estudo do preconceito e da discriminação racial a exemplo das representações e dos valores atribuídos a negros, mulatos e brancos. Ela tinha experiência não apenas na utilização de técnicas psicológicas, dentre as quais se destacava o psicodiagnóstico de Rorschach, como em pesquisas envolvendo atitudes raciais ${ }^{1}$ com base em metodologia quantitativa.

O trabalho de Ginsberg esteve associado a várias instituições educacionais e de pesquisa de caráter modernizante que desenvolviam investigações e aplicações de testes psicológicos, a saber: Escola Livre de Sociologia e Política, Instituto de Organização Racional do Trabalho (Idort), Serviço Nacional de Aprendizagem Industrial (Senai), em São Paulo, Instituto de Seleção e Orientação Profissional (Isop), no Rio de Janeiro e Instituto de Psicologia Experimental da Pontifícia Universidade Católica de São Paulo - PUC-SP.

Este artigo aborda um conjunto de trabalhos realizados por Ginsberg sobre as relações étnico-raciais no Brasil. Procuro demonstrar que o processo de inflexão da visão de Ginsberg, centrado inicialmente em investigações de natureza comportamental, passa gradativamente a mobilizar argumentos socioantropológicos para o entendimento das assimetrias raciais. Argumento que tal mudança decorreu sobretudo da influência dos estudos do psicólogo social e antropólogo Otto Klineberg, da Universidade de Columbia, ex-aluno de Franz Boas, professor do Departamento de Psicologia da Universidade de São Paulo - USP entre 1945 e 1947, e severo crítico dos testes de inteligência e do valor heurístico do conceito de

${ }^{1}$ As pesquisas sobre atitudes raciais (preconceitos e estereótipos) enfatizavam as críticas ao determinismo biológico, ao suposto caráter inato das ações humanas, buscando as razões psicossociológicas das hostilidades entre grupos sociais - étnicos, religiosos, econômicos etc. (Klineberg, 1940, pp. 346-347). Robert Park concebe as atitudes a partir de motivações econômicas, religiosas, de busca de status ou suscitadas pela discriminação a minorias, entre outras (Park, 1931, p. 31). 
raça. Ele esteve no centro da luta contra as políticas eugênicas nos EUA desde os anos 1920 e, no cenário internacional, engajou-se na definição e implementação de uma agenda antirracista, que incluiu uma pesquisa de Aniela Ginsberg, a partir do trabalho realizado na Unesco no pós-Segunda Guerra Mundial (Ginsberg, 1955; Maio, 1998).

\section{Primeiros passos de Aniela Ginsberg}

Aniela Meyer Ginsberg (1902-1986) nasceu na Polônia. Graduou-se em Psicologia, fez mestrado em Filosofia e doutorado em Psicologia pela Universidade de Varsóvia. Estudou em Berlim e Hamburgo, onde teve como professores Max Wertheimer e Wolfgang Köler, formuladores da psicologia da Gestalt; William Louis Stern, expoente da psicologia diferencial e aplicada e Kurt Lewin, que se dedicou à análise dos problemas de mudança cultural, conflito, moral e minorias (Azevedo, 2002).

Ao chegar ao Brasil em 1936, ${ }^{2}$ Ginsberg inicia seu trabalho como assistente no Laboratório de Psicologia Educacional da prestigiosa Escola Normal Caetano de Campos, em São Paulo, instituição à qual estiveram associados psicólogos e educadores ligados à reforma do ensino público brasileiro, como Lourenço Filho. Ginsberg colaborou em atividades de pesquisa com Noemy da Silveira Rudolfer, para quem a aplicação de testes psicológicos a grupos escolares constituía importante recurso na formulação de diretrizes pedagógicas mais eficientes para o processo de aprendizagem (Moraes, 2012).

Em 1937, Ginsberg tornou-se assistente de Rudolfer na cadeira de Psicologia Social na Escola Livre de Sociologia e Política (ELSP), instituição fundada em 1933 por um grupo de industriais, intelectuais e profissionais, que tinham por objetivo formar elites técnicas modernas voltadas às questões sociais e de administração pública. Os primórdios da ELSP foram pautados pelas ideias de racionalidade, pragmatismo, de aplicabilidade do conhecimento sociológico (Limongi, 1989). A ELSP manteve estreitos laços com o Instituto de Organização Racional do Trabalho (Idort), no qual Ginsberg começou a trabalhar em 1941 como diretora do Centro de Orientação Profissional (Ginsberg, 1947, p. 95; Azevedo, 2002).

${ }^{2}$ Em 1928, Aniela Ginsberg casou-se com o economista Tadeuz Wladyslaw Ginsberg, tendo vivido na Itália por dois anos. Em 1936, ele foi contratado por uma instituição financeira de São Paulo (Puppo, 2010). 
A experiência de trabalho com testes psicológicos conduziu Ginsberg ao Departamento do Serviço Nacional de Aprendizagem Industrial (Senai) de São Paulo, onde trabalhou na Divisão de Seleção e Orientação Profissional (1943-1946). Suas investigações envolveram a validação de instrumentos que permitiram a medição de fenômenos psicológicos para os serviços de orientação educacional, profissional e seleção de pessoal, dentre os quais figuravam testes de inteligência, bem como a condução de estudos de caso, tendo em vista a orientação psicopedagógica de jovens aprendizes matriculados nos cursos de formação profissional do Senai.

\section{A análise dos grupos raciais pela psicologia diferencial}

As pesquisas de Aniela Ginsberg sobre grupos étnico-raciais foram realizadas em duas frentes. Na primeira, a psicóloga fixou-se no estudo das possíveis diferenças psíquicas, comportamentais e cognitivas entre indivíduos classificados nesses distintos segmentos (Ginsberg, 1953). Na outra frente, valendo-se da psicologia social e de conceitos como o de atitude, Ginsberg não apenas abordou o preconceito racial, como também se esforçou por avaliá-lo estatisticamente entre crianças e jovens brasileiros de diferentes grupos étnico-raciais e estratos sociais. A agenda de pesquisa que se entrevê nos estudos de Ginsberg se desdobra em dois conjuntos de problemas: 1) a existência ou não de características raciais inatas, como a capacidade mental; 2) a natureza das relações raciais no contexto brasileiro a partir do estudo das atitudes de preferência e rejeição entre os indivíduos com base na cor.

No plano da psicologia diferencial, ${ }^{3}$ a primeira pesquisa de Ginsberg, quando lecionava na Faculdade de Medicina da Bahia, ocorreu entre 1946 e 1947, contemplando a avaliação de cem crianças de escolas públicas de Salvador classificadas em diferentes grupos raciais, mediante o teste de Rorschach. ${ }^{4}$ Segundo Ginsberg, o Estado da Bahia representaria o Brasil da mistura racial, lembrando a tradicional visão sobre as relações raciais no país, ao conceber o encontro

${ }^{3}$ No Brasil, os estudos sobre diferenças individuais de aptidão mental, principalmente no caso da aplicação de testes de inteligência no setor educacional, eram reunidos sob a rubrica de "psicologia diferencial” (Dávila, 2003, p. 141).

${ }^{4}$ A técnica foi desenvolvida pelo psiquiatra suíço Hermann Rorschach. As lâminas, contendo os borrões de tinta, obedecem a características específicas quanto à proporção, à angularidade, à luminosidade, ao equilíbrio espacial e às cores. A partir das respostas, procura-se obter um quadro amplo da dinâmica psicológica do indivíduo (Nascimento, 2002). 
harmonioso dos grupos étnico-raciais que "fala[vam] a mesma língua, cresc[iam] no mesmo meio físico, geográfico e social [e] frequenta[vam] a mesma escola" (Ginsberg, 1950, mar., p. 32). A mistura, nesse caso, referia-se não apenas ao fenômeno da miscigenação, mas também à ideia de que negros, brancos e mulatos viviam sob as mesmas condições sociais, de modo que qualquer diferença nos resultados do teste não expressaria a influência do meio social. Ginsberg buscava, desse modo, avaliar a atuação isolada de "fatores raciais" na produção de diferenças na personalidade (pp. 2-3).

Com base em traços fenotípicos (cor da pele, textura do cabelo, forma do nariz e dos lábios), tradicional modelo de identificação dos indivíduos inspirado na antropologia física, os escolares foram classificados como "brancos" ou "quase brancos" e "pretos" ou "quase pretos", categorias elásticas utilizadas por Ginsberg para capturar as nuanças de uma sociedade percebida como intensamente miscigenada. Em seguida, para fins de comparação, os estudantes foram dispostos em pares que deviam ser do mesmo sexo e apresentar idade e rendimento escolar semelhantes. A fim de garantir que todas as crianças fossem das mesmas classes sociais, Ginsberg restringiu a pesquisa a alunos vinculados às camadas populares e à classe média, uma vez que somente na rede pública de ensino era possível encontrar um número suficiente de indivíduos pertencentes a ambos os grupos raciais sob estudo, brancos e negros (p. 4).

Após analisar as respostas ao teste de Rorschach ${ }^{5}$ e submetê-las a tratamento estatístico, Ginsberg forneceu características gerais do grupo de jovens examinado, cujo nível mental parecia estar abaixo das médias obtidas em avaliações realizadas por outros pesquisadores com diferentes populações no Brasil e no exterior. ${ }^{6}$ Em média, os jovens indicaram "uma mentalidade mais prática que teórica". No tocante às diferenças entre crianças brancas e negras, Ginsberg observou que as primeiras apresentaram "nível mental superior", "mentalidade mais teórica" e menor "inibição afetiva", enquanto as últimas demonstraram "mentalidade mais prática" (p. 31). Ao apresentar tais conclusões, que apontavam para a inferioridade intelectual das crianças negras, Ginsberg mostrava-se cautelosa, afirmando que os resultados eram estatisticamente pouco significativos.

${ }^{5}$ Nesta técnica de avaliação psicológica, dez lâminas com borrões de tinta são apresentadas ao indivíduo a fím de que forneça interpretações para cada uma delas. Trata-se de uma técnica projetiva, ou seja, supõe-se que a pessoa "projete" sua personalidade nas manchas de tinta ao interpretá-las (Nascimento, 2002).

${ }^{6}$ Ginsberg cita, entre outros, Luiz Cerqueira, Behn-Eschenburg, Mary Ford, Margarite Hertz, Lopfe A., Loosli-Usteri, Kerr e Serebrinsky (Ginsberg, 1950 mar., p. 32) Ver também Rocha (2011) para os casos de Isaias Alves, Anísio Teixeira e Lourenço Filho. 


\section{HISTÓRIA DA PSIQUIATRIA}

Nesse caso, ela destacava a necessidade de conduzir novas pesquisas sobre a diferença de nível mental entre negros e brancos que levasse em consideração as condições socioeconômicas (Ginsberg, 1951, p. 28).

A pesquisa na Bahia teve desdobramentos em fins dos anos 1940, período em que Ginsberg trabalhou no Instituto de Seleção e Orientação Profissional (Isop), no Rio de Janeiro. A psicóloga teve acesso aos resultados de um teste de inteligência aplicado entre 1947 e 1948 por técnicos do Departamento Nacional da Criança (DNCr), cuja população-alvo era formada por adolescentes de escolas secundárias de algumas capitais brasileiras.

Para fins de comparação, os alunos foram classificados por sexo, idade, classe social e traços fenotípicos. Devido ao pequeno número de alunos negros e mulatos pertencentes aos segmentos sociais dominantes, foram comparados somente os resultados dos indivíduos que faziam parte das classes média e inferior. Ao trabalhar com provas de nível mental entre escolares de diferentes grupos étnico-raciais, Ginsberg dava prosseguimento à investigação conduzida na Bahia a partir do psicodiagnóstico de Rorschach. O escopo da nova pesquisa, no entanto, era mais amplo, uma vez que se tratava de examinar diferenças de ordem cognitiva conforme os diferentes fatores acima mencionados.

Ao confrontar as notas obtidas pelos alunos, Ginsberg concluiu que os resultados médios tendiam a piorar nas classes baixas, de forma que os melhores resultados se concentravam no grupo de jovens filhos de profissionais liberais. Ademais, em grandes cidades como o Rio de Janeiro, cuja média dos resultados era melhor, o desempenho de alunos mulatos e negros era superior ao de alunos brancos de cidades menores, como Fortaleza. Estes resultados, segundo Ginsberg, aproximavam-se daqueles obtidos por Klineberg a partir de testes de inteligência aplicados a negros e brancos nos Estados Unidos. Nesse caso, os escolares negros na região Norte, mais desenvolvida, haviam apresentado resultados superiores aos brancos vivendo em estados do Sul (Ginsberg, 1951, pp. 41-42).

Todavia, Ginsberg pondera que no interior das classes sociais consideradas pela pesquisa do Departamento Nacional da Criança, as diferenças observadas entre as notas de alunos brancos, negros e índios, apontavam para a "inferioridade relativa dos pretos". Diante das possíveis causas deste fenômeno, Ginsberg assume posição reticente, afirmando que ele não dependia "diretamente do meio nem da hereditariedade" (p. 41).

Com os resultados da pesquisa sobre nível mental e personalidade entre negros e brancos, Ginsberg assumiu uma perspectiva cética quanto às possibilidades de estudo de características raciais inatas, das diferenças individuais ligadas à variável "grupo racial". A psicóloga passa a criticar a validade dos testes de inteligência que apresentavam um viés "etnocêntrico" e de classe, na esteira de Klineberg (Ginsberg, 1951, 1953, 1953 dez.). 


\section{A abordagem socioantropológica sobre preconceito e discriminação}

Em artigo publicado na coletânea organizada por Klineberg, Ginsberg (1953) observa que "as diferenças educacionais e socioeconômicas [entre negros e brancos] podiam invalidar completamente os resultados de uma comparação entre grupos raciais" (p. 230). Comparações psicológicas com base em "raças" estavam sujeitas a "tantas influências do meio socioeconômico e cultural, que [não permitiam ao estudioso] tirar nenhuma conclusão sobre as aptidões inatas" (p. 232). Nas palavras de Ginsberg, até então "os estudos psicológicos não [haviam conseguido] demonstrar a existência de diferenças raciais inatas, independentes das influências do meio" (p. 232).

A visão crítica dos estudos de Klineberg em relação aos testes de inteligência constituíam importante referência para Ginsberg, principalmente Characteristics of the American Negro (1944), livro organizado pelo psicólogo canadense que reuniu os trabalhos de natureza interdisciplinar elaborados no âmbito da ampla pesquisa coordenada por Gunnar Myrdal, e que redundou no An American Dilemma (1944), um clássico sobre os fundamentos do racismo nos Estados Unidos. Segundo Ginsberg (1953), as pesquisas de Klineberg haviam chamado a atenção para a significativa influência do ambiente educacional sobre os resultados dos testes (p. 230).

Os testes continham ainda um viés cultural por terem sido construídos segundo normas, valores e expectativas de grupos específicos da sociedade americana. Seu posicionamento está calcado nos resultados das pesquisas conduzidas por Klineberg entre crianças da comunidade indígena dos Yakima (Toppenish, Washington), de brancos da cidade de Nova York e de negros do estado da Virgínia, revelando que as crianças indígenas não tinham motivações culturais semelhantes às das crianças norte-americanas no tocante aos resultados da aplicação de testes. A exigência de que respondessem rápido às questões, por exemplo, era-lhes destituída de significado, ao passo que as crianças brancas eram instadas desde cedo, a partir do ambiente escolar, à realização de tarefas no menor tempo possível. Neste caso, os testes pareciam medir hábitos intelectuais desenvolvidos com a cultura do grupo, e não atributos cognitivos inatos (Klineberg, 1928; 1935; Ginsberg, 1953, pp. 230-231).

Nesta fase, os trabalhos de Ginsberg concentram-se na questão da distribuição de características psicológicas entre os indivíduos conforme critérios étnico-raciais e de classe. Eles também indicam o interesse científico que as reflexões de Klineberg sobre a validade dos testes psicológicos haviam despertado em Ginsberg que passou a acentuar a influência de fatores socioeconômicos e culturais no desempenho intelectual dos jovens e das crianças. 


\section{HISTÓRIA DA PSIQUIATRIA}

A primeira investigação de Aniela Ginsberg envolvendo preconceito racial foi inspirada nos estudos de Klineberg. Realizada entre 1946 e 1947, com 723 alunos de escolas públicas de Salvador de 6 a 20 anos, a pesquisa tinha por objetivo verificar se as variáveis idade, sexo e raça influíam na escolha, pelo aluno, do seu companheiro de carteira. Neste sentido, os alunos eram indagados sobre quais seriam a primeira e a segunda opções caso pudessem escolher. Aos mais velhos também se perguntavam as razões da escolha. Ginsberg tomou como referência o método sociométrico elaborado por Jacob Moreno, cujo objetivo era sondar as atitudes raciais das crianças sem confrontá-las diretamente com perguntas sobre preconceito racial, na medida em que poderiam comprometer a espontaneidade das respostas (Ginsberg, 1947a, pp. 3-4).

Seguindo indicação de Klineberg, Ginsberg classificou os estudantes em "completamente brancos" (20,05\%), "não completamente brancos" $(28,22 \%)$, "mulatos" (24,6\%), "não completamente pretos" (18,25\%) e "completamente pretos" $(9,41 \%)$. Tal critério, mais uma vez, teve por base a aparência racial e as características físicas (cor da pele, tipo de cabelo, o formato do nariz e dos lábios, a qualidade do cabelo), ou seja, nas características fenotípicas dos indivíduos (p. 5). ${ }^{7} \mathrm{O}$ sistema classificatório abrangente e impreciso proposto por Klineberg, constituído por um gradiente de categorias fluidas, se coadunava com uma percepção da situação racial baiana que enfatizava a mistura, a indeterminação, a subjetividade e a dependência contextual de sua aplicação.

Ginsberg observa que o grupo de alunos pesquisados não era representativo da população escolar de Salvador, uma vez que crianças pertencentes às classes média e alta baianas frequentavam majoritariamente escolas particulares não incluídas no estudo. Nas instituições privadas, "só excepcionalmente, se encontra[vam] crianças de cor", de modo que não era factível estudar as atitudes raciais nestes meios segundo o método sociométrico em questão. Desse modo, o foco da pesquisa recaiu nas escolas públicas, nas quais era possível encontrar "população bem mista" (pp. 6-8). Note-se que observações semelhantes estão presentes em outros estudos da psicóloga, como aquele envolvendo a aplicação do teste de Rorschach em escolares baianos (Ginsberg, 1950 mar.). As observações de Ginsberg revelam evidências sociológicas importantes sobre o contexto baiano, como a posição social subalterna em que negros e mulatos geralmente se encontravam.

${ }^{7}$ Ginsberg se inspira na Escala de Likert, um modelo de escala de resposta psicométrica utilizada em questionários e pesquisas de opinião. Ao responderem a um questionário baseado nessa escala, os respondentes especificam seu nível de concordância com uma afirmação (Likert, 1932). 
Para controlar o viés que pudesse influenciar as opções dos alunos, Ginsberg trabalhou com o percentual correspondente às crianças de cada cor que eram escolhidas em função do número disponível de brancos, mulatos e negros em cada turma. Com base na razão entre o número total de escolhas feitas pelas crianças de determinada cor e a ocorrência de cada grupo de cor no universo total de crianças pesquisadas, a psicóloga estabeleceu um "índice de preferência". Nesse caso, o preconceito foi definido enquanto falta de preferência por alunos de determinada cor (Ginsberg, 1947a, p. 9).

Embora as crianças escolhessem com maior frequência companheiros da mesma cor, notava-se, no número total de respostas, "uma leve preferência [pelas] crianças mais claras" e, dentre estas, pelas crianças "completamente brancas". Estas "conserva[vam] um certo "prestígio", sendo as escolhidas com maior frequência por crianças de outras cores. Por seu turno, as crianças classificadas como "completamente pretas" raramente apareciam entre as mais escolhidas, isto é, o índice de preferência diminuía conforme a elevação das diferenças de cor entre os escolares. Segundo Ginsberg (1947), estes resultados indicavam que a cor incidia nas escolhas feitas pelos alunos (pp. 9-11).

Indagadas sobre as razões de suas preferências, as crianças apontaram com maior frequência aquelas relacionadas com "amizade e gosto pessoal", seguidas por outras relativas ao bom desempenho escolar e ao auxílio do colega nos estudos. Ainda que o critério racial não fosse explicitado em suas justificativas, o preconceito fazia-se presente na forma de "falta de preferência" pelas crianças de cor, com mais frequência preteridas no processo de escolha do companheiro de carteira. Embora as crianças agissem com base no preconceito, não mencionavam problemas ligados à cor ou raça (pp. 9 e 17).

Ginsberg retomou o estudo do preconceito no ano de 1947, ao realizar um inquérito sobre atitudes de 400 jovens universitários de Salvador diante de diferentes grupos étnico-raciais e nacionalidades. Desta vez, sua investigação esteve ancorada na técnica para aferição da distância social entre indivíduos desenvolvida pelo sociólogo norte-americano Emory Bogardus. Além de um inquérito inspirado na escala de Bogardus (1933), segundo o qual os estudantes deviam classificar grupos étnico-raciais e nacionais de acordo com o grau de proximidade ou intimidade que aceitariam manter com seus membros, eles deviam indicar, se fosse o caso, o "grupo racial, nacional, religioso, político ou social" (Ginsberg, 1950 jun., p. 11).

Analisando os resultados do inquérito, Ginsberg observou que, dentre as nacionalidades preferidas para relações de parentesco, amizade ou vizinhança, destacavam-se norte-americanos, franceses, portugueses e ingleses. Os mais indicados para expulsão do país foram sobretudo os japoneses, com 45,5\% das escolhas, seguidos dos russos $(28,5 \%)$, alemães $(21,5 \%)$, judeus $(20,5 \%)$, espanhóis $(10,2 \%)$ e sírios $(6,5 \%)$. A reação negativa em face de japoneses, alemães e russos 


\section{HISTÓRIA DA PSIQUIATRIA}

devia ser entendida, segundo Ginsberg, no contexto da Segunda Guerra Mundial e, no caso dos russos em particular, no da propaganda anticomunista (p. 12).

No tocante às atitudes diante de grupos étnicos, a psicóloga notou um acentuado antissemitismo por parte dos entrevistados. Os judeus foram raramente aceitos como membros da família, com apenas $1,2 \%$ das escolhas nesta categoria, sendo associados com mais frequência aos grupos a serem "expulsos do país". Os negros também foram apontados como possíveis membros da família somente em 1,2\% das escolhas. Aparecem, com mais assiduidade, como "amigos" e "colegas", em categorias em que obtiveram, respectivamente, $5,5 \%$ e 1,7\% do total de escolhas; percentuais baixos se comparados a outros grupos. Para Ginsberg, a baixa frequência com que negros e mulatos haviam sido mencionados - isto é, apenas 72 e 78 vezes, respectivamente, no total de 400 respostas - significava que os "informantes [pareciam] fugir do problema da cor" (p. 12). Ginsberg chegava a resultados semelhantes aos da pesquisa com alunos de escolas públicas ("Escolha do companheiro de carteira - Resultado de um inquérito entre escolares baianos") ao observar que os jovens eram reticentes em relação à questão da cor, e que os negros eram habitualmente preteridos ou ignorados no estabelecimento das relações de proximidade. Na opinião dos entrevistados acerca de grupos que constituíam um problema na Bahia, sobressaíram "comunistas", "negros" e "judeus", com respectivamente $17,34 \%, 15,30 \%$ e $15,30 \%$ do total de escolhas. Segundo Ginsberg, as respostas a esta questão pareciam evidenciar as tendências reveladas pelo questionário de Bogardus (p. 15).

\section{Finalizando: miscigenação, preconceito e o projeto Unesco}

Ginsberg atribuía a interação social no contexto escolar brasileiro, em que crianças negras, brancas e mulatas "brincavam juntas", um caráter predominantemente inclusivo em matéria racial. No entanto, suas investigações indicaram que essa miscigenação parecia se restringir aos alunos da rede pública de ensino, pertencentes, em sua maioria, às classes subalternas, e que mesmo as relações entre esses indivíduos não eram destituídas de discriminação e preconceito.

Os achados de Ginsberg ficam ainda mais evidentes no estudo realizado no âmbito do ciclo de pesquisas sob a chancela da Unesco nos anos 1950 (Maio, 1999), no contexto de uma série de iniciativas educacionais de combate ao racismo. Indicada por Otto Klineberg, coordenador do projeto Tensions Affecting International Understanding, patrocinado pela agência internacional, Ginsberg retornou ao problema das atitudes raciais, desta vez trabalhando com 208 crianças em idade escolar na cidade de São Paulo. Ao definir seu objeto de pesquisa, ela 
reiterou sua visão de que o Brasil era um país de acentuada "mistura de raças", onde conviviam diferentes grupos étnico-raciais: observa "crianças brancas, amarelas, mulatas e pretas irem à aula e brincarem juntas". Considera, contudo, a existência do "preconceito de raça" no país e tem como objetivo de pesquisa avaliar as formas de manifestação do fenômeno social e sua frequência. Ginsberg (1955) elegeu para este fim, a questão das atitudes de escolares em relação a colegas brancos, mulatos e pretos, buscando verificar, mais precisamente, em que medida uns demonstravam atitudes hostis para com os outros.

O resultado da pesquisa sugere que, embora o "preconceito de raça" não se manifestasse em atitudes declaradamente hostis, como na verbalização de antagonismos, ele se fazia presente em diferentes meios sociais, sendo mais frequente e acentuado entre os estratos sociais superiores. O preconceito era identificável sobretudo na generalizada preferência das crianças e dos jovens examinados por indivíduos brancos em detrimento da população de cor, o mesmo ocorrendo no estabelecimento das relações íntimas de amizade ou parentesco, como no caso dos padrões ideais de beleza que se perseguiam e cuja referência, inclusive para negros e mulatos, eram os brancos. Ao destacarem os papéis sociais subalternos e a condição econômica inferior que eram atribuídos com frequência aos negros pelas crianças, as pesquisas de Ginsberg incidiam sobre o tema sociológico das desigualdades, chamando a atenção para o lugar desses indivíduos não apenas nas representações, mas também na própria estrutura social. Elas indicavam que a população negra enfrentava barreiras de cor e classe, lembrando as análises de outros estudos do projeto Unesco como os de Virgínia Bicudo, Oracy Nogueira e Luiz de Aguiar Costa Pinto.

\section{Referências}

Azevedo, M.L.B. de, \& Ginsberg, A.M. (2002). In R.H.de D. Campos (Org.). Dicionário Biográfico da Psicologia no Brasil: pioneiros (pp. 166-171). Rio de Janeiro: Imago.

Bogardus, E. (1933). A Social Distance. Sociology and Social Research, 17, 265-271.

Dávila, J. (2003). Diploma of Whiteness: Race and Social Policy in Brazil, 1917-1945. Durham: Duke University Press.

Ginsberg, A. (1947). Métodos projetivos. Boletim Bibliográfico. Publicação da Biblioteca Pública Municipal de São Paulo, Departamento de Cultura, X, 95-105.

Ginsberg, A. (1947a). Escolha do companheiro de carteira - Resultado de um inquérito entre escolares baianos. Psyke: Revista didática e cientifica de Psicologia, Psiquiatria e Psicanálise, Rio de Janeiro, I(3), 3-17. 


\section{HISTÓRIA DA PSIQUIATRIA}

Ginsberg, A. (1950, mar.). Um estudo de 100 jovens baianos, com o teste de Rorschach. Neurobiologia, 1, 1-50.

Ginsberg, A. (1950, jun.). Um inquérito sobre as atitudes de estudantes baianos em relação a diversos grupos nacionais e raciais. Boletim de Psicologia, São Paulo, I(4), 10-15.

Ginsberg, A. (1951, dez.). Comparação entre os resultados de um teste de nível mental aplicado em diferentes grupos étnicos e sociais. Arquivos Brasileiros de Psicotécnica, IV(4), 27-44.

Ginsberg, A. (1953). Psicologia diferencial. In O. Klineberg (Org.). A psicologia moderna (pp. 204-237). São Paulo: Agir.

Ginsberg, A. (1953, dez.). Estudo comparativo dos interesses de adolescentes de diferentes meios sociais. Arquivos Brasileiros de Psicotécnica, V(4), 7-31.

Ginsberg, A. (1955). Pesquisas sobre as atitudes de um grupo de escolares de São Paulo em relação às crianças de cor. In R. Bastide, \& F. Fernandes. Relações raciais entre negros e brancos em São Paulo (pp. 311-361). São Paulo: Anhembi/Unesco.

Klineberg O. (1928). An experimental study of speed and other factors in "racial" differences. Archives of Psychology, 93, 1-111.

Klineberg O. (1935). Race Differences. New York: Harper.

Klineberg, O. (1940). Social Psychology. New York: Henry Holt and Company. pp. 345-346 .

Klineberg O. (1944). Characteristics of the American Negro. New York and Evanston: Harper \& Brothers.

Klineberg O. (1953). Psicologia social. In O. Klineberg (Org.). A psicologia moderna (pp. 132-156). São Paulo: Agir.

Likert, R. (1932). A technique for the measurement of attitudes, Archives of Psychology 140, 1-55.

Limongi, F. (1989). A Escola Livre de Sociologia e Política de São Paulo. In S. Miceli (Org.). História das Ciências Sociais no Brasil. (Vol. 1, pp. 217-233). São Paulo: Vértice/Idesp/ Finep.

Maio, M.C. (1998). O Brasil no concerto das nações: a luta contra o racismo nos primórdios da Unesco. História, Ciências, Saúde-Manguinhos, 5(2), 375-413.

Maio, M.C. (1999). O Projeto Unesco e a agenda das ciências sociais no Brasil dos anos 40 e 50. Revista Brasileira de Ciências Sociais, 14(41), 141-158.

Maio, M.C. (2010). Educação sanitária, estudos de atitudes raciais e psicanálise na trajetória de Virgínia Leone Bicudo. Cadernos PAGU, 35, 309-355.

Moraes, J.D. de. (2012, abr./jun.). Noemy Rudolfer e a organização da escola e do mundo do trabalho nos anos 1920 e 1930. Educação e Pesquisa, São Paulo, 36(2), 485-497.

Nascimento, S.R.G. (2002). Estudo normativo do sistema compreensivo do Rorschach para a cidade de São Paulo. PsicoUSF [online], 7(2), 127-141. 
Park, R.E. (1931). Human Nature, Attitudes and the Mores. In K. Young, Social Attitudes (pp. 17-45). New York: Henry Holt.

Pupo, N.C. (2010). Aniela Meyer Ginsberg. In H.C.Costa Boa-Viagem (Org.). Elas vieram de Longe... Séculos XIX e XX (pp. 239-247). São Paulo: Scortecci.

Rocha, A.C.S.M. (2011). O que fazer com os rudes? Isaías Alves e as divergências sobre o papel da inteligência na organização escolar (1930-1942). Dissertação de Mestrado em História, Política e Bens Culturais, CPDOC-FGV, Rio de Janeiro, 2011.

\section{Resumos}

(Measuring racial prejudice in Brazil: Aniela Ginsberg and the study of racial atitudes)

This article analyzes a series of studies by Ginsberg on race relations in Brazil. It tries to describe the inflection process of the vision of a psychologist who, based on behavioral research, started using socio-anthropological arguments for the understanding of racial disparities. This change was caused by the influence of the studies of social psychologist and anthropologist Otto Klineberg, Columbia University, a former advisee of Franz Boas and a severe critic of intelligence tests and the heuristic value of the race concept.

Keywords: Aniela Ginsberg, racism, intelligence tests, racial attitudes

(Mesurer les préjugés raciaux au Brésil: Aniela Ginsberg et l'étude des attitudes raciales)

Cet article analyse une série d'études menées par Ginsberg sur les rapports entre les races au Brésil. Il illustre le processus de transformation du point-de-vue de la psychologue qui, en conduisant des recherches de nature comportementale, passe à utiliser des arguments socio-anthropologiques pour expliquer les différences entre les races. Ce changement est dù à l'influence des études du psychologue social et anthropologue Otto Klineberg de l'Université de Columbia, doctorant de Franz Boas et critique sévère des tests d'intelligence et de la valeur heuristique de la notion de race. Mots clés: Aniela Ginsberg, le racisme, tests d'intelligence, attitudes raciales

(Mediendo el prejuicio racial en Brasil: Aniela Ginsberg y el estudio de las actitudes raciales)

En este artículo se analizan una serie de estudios llevados a cabo por Ginsberg acerca de las relaciones interraciales en Brasil. Trata de mostrar el proceso de transformación de la visión de la psicología que, basado en investigaciones del comportamiento, va a emplear argumentos socio-antropológicos para la 


\section{HISTÓRIA DA PSIQUIATRIA}

comprensión de las desigualdades raciales. Dicho cambio se debió a la influencia de los estudios del psicólogo social y antropólogo Otto Klineberg, de la Universidad de Columbia, antiguo alumno de Franz Boas y crítico severo de las pruebas de inteligencia y del valor heurístico del concepto de raza.

Palabras clave: Aniela Ginsberg, el racismo, pruebas de inteligencia, actitudes raciales

(Die Einstufung rassistischer Vorurteile in Brasilien: Aniela Ginsberg und das Studium der Rassen Einstellungen)

Dieser Artikel untersucht eine Reihe von Studien, die von Ginsberg in Bezug auf Rassenbeziehungen in Brasilien durchgeführt wurden. Er versucht, den Wandlungsprozess der Auffassung der Psychologin darzustellen, welche sich auf der Verhaltensforschung basierte Studien stützte, um sozio-anthropologische Argumente für das Verständnis der Rassenunterschiede anzuwenden. Diese Wandlung war das Resultat des Einflusses von Studien des Sozialpsychologen und Anthropologen Otto Klineberg von der Columbia University, dessen Doktorvater Franz Boas war, und welcher ein scharfer Kritiker von Intelligenztests und des heuristischen Wertes des Rassenkonzepts war.

Schlüsselwörter: Aniela Ginsberg; Rassismus; Intelligenztests; rassistische Einstellung

（测量在巴西的种族歧视：Aniela Ginsberg和种族态度的研究）

本文分析了一系列Aniela Ginsberg在巴西的种族关系的研究。试图表明 此心理学家在研究时从行为分析开始通过社会人类学来了解种族的不平衡。此 变化主要来自于0tto Klineberg, 哥伦比亚大学的社会心理和人类学家, Franz Boas的学生, 而一名严重批评智力测试和种族概念的启发价值。

关键词: Aniela Ginsberg, 种族主义, 智力测验, 种族态度

Citação/Citation: Maio, M.C. (2015, dezembro). Medindo o preconceito racial no Brasil: Aniela Ginsberg e o estudo das atitudes raciais. Revista Latinoamericana de Psicopatologia Fundamental, 18(4), 728-742.

Editores do artigo/Editors: Profa. Dra. Ana Maria G. Raimundo Oda e Prof. Dr. Paulo Dalgalarrondo 
Recebido/Received: 5.8.2015/ 8.5.2015 Aceito/Accepted: 8.11.2015 / 11.8.2015

Copyright: (C) 2009 Associação Universitária de Pesquisa em Psicopatologia Fundamental/ University Association for Research in Fundamental Psychopathology. Este é um artigo de livre acesso, que permite uso irrestrito, distribuição e reprodução em qualquer meio, desde que o autor e a fonte sejam citados / This is an open-access article, which permits unrestricted use, distribution, and reproduction in any medium, provided the original authors and sources are credited.

Financiamento/Funding: Pesquisa financiada pelo Conselho Nacional de Desenvolvimento Científico e Tecnológico - CNPq (Processo n. 311895/2014-0) / Research funded by the Conselho Nacional de Desenvolvimento Científico e Tecnológico - CNPq (Processo n. 311895/2014-0).

Conflito de interesses/Conflict of interest: $\mathrm{O}$ autor declara que não há conflito de interesses / The author has no conflict of interest to declare.

\section{Marcos Chor Maio}

Pesquisador titular; Professor do Programa de Pós-graduação em História das Ciências e da Saúde da Casa de Oswaldo Cruz/Fiocruz (Rio de Janeiro, RJ, Br); Bolsista de Produtividade em Pesquisa do CNPq 1B (Brasília, DF, Br).

Rua Visconde de Pirajá, 592/503

22410-002 Rio de Janeiro, RJ

e-mail:maio@fiocruz.br

This is an open-access article, which permits unrestricted use, distribution, and reproduction in any medium for non-commercial purposes provided the original authors and sources are credited. 